\title{
The use of trastuzumab affected by health insurance policy in Jiangsu Province of China
}

\author{
Yiqin Xia ${ }^{1 \#}$, Mingjie Zheng ${ }^{1 \#}$, Xiang Zhan ${ }^{2 \#}$, Ying Liu ${ }^{3}$, Susheng Cao ${ }^{4}$, Qing Shao ${ }^{5}$, Dong Meng ${ }^{6}$, \\ Liyan Jin ${ }^{7}$, Lingyun $\mathrm{Xu}^{8}$, Tongbo Yi ${ }^{9}$, Hui Xie ${ }^{1}$, Shui Wang ${ }^{1}$
}

${ }^{1}$ Department of Breast Surgery, The First Affiliated Hospital, Nanjing Medical University, Nanjing, China; ${ }^{2}$ School of Health Policy and Management, Nanjing Medical University, Nanjing, China; ${ }^{3}$ Institude of Medical Education, Nanjing Medical University, Nanjing, China; ${ }^{4}$ Department of Breast Surgery, Xuzhou Central Hospital, The Affiliated Xuzhou Hospital of Medical College of Southeast University, Xuzhou, China; ${ }^{5}$ Department of Thyroid and Breast Surgery, Affiliated Jiangyin Hospital of Southeast University, Jiangyin, China; ${ }^{6}$ Department of Oncological Surgery, The Affiliated Hospital of Jiangnan University, Wuxi, China; ${ }^{7}$ Department of General Surgery, The Second Affiliated Hospital of Soochow University, Suzhou, China; ${ }^{8}$ Department of Breast Surgery, The Second Affiliated Changzhou People's Hospital of Nanjing Medical University, Changzhou, China; ${ }^{9}$ Department of Thyroid and Breast Surgery, Jiangsu Taizhou People's Hospital, Taizhou, China

Contributions: (I) Conception and design: Y Xia, H Xie, S Wang; (II) Administrative support: H Xie, S Wang; (III) Provision of study materials or patients: Y Xia, M Zheng, X Zhan, S Cao, Q Shao, D Meng, L Jin, L Xu, T Yi; (IV) Collection and assembly of data: Y Xia, M Zheng, X Zhan, Y Liu; (V) Data analysis and interpretation: Y Xia, M Zheng, X Zhan, Y Liu; (VI) Manuscript writing: All authors; (VII) Final approval of manuscript: All authors.

"These authors contributed to this work equally

Correspondence to: Hui Xie; Shui Wang. Department of Breast Surgery, The First Affiliated Hospital, Nanjing Medical University, Nanjing 210029, China. Email: hxie@njmu.edu.cn; ws0801@hotmail.com.

Background: Breast cancer recurrence and mortality have been shown to decrease after trastuzumab treatment in human epidermal growth factor 2 (HER2)-positive early-stage breast cancer (EBC) patients. In Jiangsu Province, trastuzumab has been subsidized for patients with HER2-positive EBC since 2013. Several studies showed that Jiangsu was one of the provinces with the highest rates of adjuvant trastuzumab therapy. To uncover the underlying reason, we designed the study to investigate trastuzumab use for HER2-positive breast cancer patients, and to examine the changes caused by medical insurance coverage for trastuzumab in Jiangsu province of China.

Methods: This was a retrospective, multicenter clinical study with follow-up data. HER2-positive EBC patients diagnosed in 7 representative hospitals in 2010, 2011, and 2013 were enrolled. Demographic and clinical data, and details of diagnosis, treatments, and prognosis, were collected. Data analysis included univariate analysis, multivariate logistic regression, survival analysis, and subgroup analysis.

Results: Of the 641 patients (mean age $51.01 \pm 10.79$ years) included, $412(64.27 \%)$ patients had medical insurance. Trastuzumab therapy was given to 214 (33.39\%) patients. The multivariate logistic regression showed that medical insurance coverage, age, and radiotherapy were associated with trastuzumab use $(\mathrm{P}<0.05)$. The overall survival was significantly better in the trastuzumab group than in the non-trastuzumab group (HR: 1.607; 95\% CI: 1.046-2.469; $\mathrm{P}=0.040$ ). Subgroup analysis revealed that there was a trend towards more patients with medical insurance $(\mathrm{P}=0.073)$, and significantly more patients received trastuzumab therapy $(\mathrm{P}<0.001)$ in 2013 than in 2010-2011. Additionally, trastuzumab use in China was lower than in developed countries. Patients with medical insurance were more likely to use trastuzumab, and more patients could afford trastuzumab therapy with the development of China's health-care reform.

Conclusions: Our study suggested that the percentage of patients who received trastuzumab in China was lower than developed countries. Patients who had medical insurance were more likely to use trastuzumab than those without medical insurance. The health insurance policy in China has improved access for breast cancer patients who require trastuzumab therapy.

Keywords: Health insurance; breast cancer; trastuzumab 
Submitted Nov 09, 2020. Accepted for publication Jan 14, 2021.

doi: $10.21037 /$ tcr-20-3329

View this article at: http://dx.doi.org/10.21037/tcr-20-3329

\section{Introduction}

Breast cancer is the most commonly diagnosed cancer $(11.6 \%)$ and the leading cause of cancer death $(6.6 \%)$ among females worldwide (1). Different subtypes of breast cancer have distinct risk profiles and prognosis (2). Approximately 20\% of breast cancer patients are human epidermal growth factor 2 (HER2)-positive, which was known to have a poorer prognosis before the development of targeted therapies (3).

Trastuzumab (Herceptin, Roche), a monoclonal antibody targeting the extracellular domain of ERBB2, is a targeted therapy for HER2-positive breast cancer patients. Breast cancer recurrence and mortality have been shown to decrease after trastuzumab treatment in HER2-positive early-stage breast cancer (EBC) patients (4). Clinical guidelines recommend trastuzumab-based chemotherapy (along with pertuzumab in some cases) as the standard (neo) adjuvant therapy for HER2-positive EBC patients (5-7).

Trastuzumab was approved by the US Food and Drug Administration (FDA) and the European Medicines Agency (EMA) as an adjuvant therapy in 2006, and by the EMA as a neoadjuvant therapy in 2011 for patients with HER2-positive EBC (8). Trastuzumab was registered by the China Food and Drug Administration (now renamed State Administration for Market Regulation) in 2008 as the treatment for HER2-positive EBC patients. In 2009, China launched a major health-care reform (9). Under this new system, since January 2013, trastuzumab has been subsidized for patients with HER2-positive EBC under the Ministry of Human Resources and Social Security of the People's Republic of China (MOHRSS). The policy has greatly reduced the out-of-pocket costs of patients with HER2-positive EBC. Jiangsu was one of the first provinces to reimburse trastuzumab for patients, so the data regarding the use of trastuzumab affected by health insurance in Jiangsu is most representative.

Two studies have estimated that approximately $40.5 \%$ (10) and $29.8 \%$ (11) of patients with HER2-positive EBC received trastuzumab therapy in China. Jiangsu was one of the provinces with the highest rates of adjuvant trastuzumab therapy (11). Since both studies were conducted after
2012, we assumed that medical insurance coverage for trastuzumab in Jiangsu contributed to its widespread use. Therefore, the current study aims to investigate the current use of trastuzumab in patients with EBC, and to examine the changes caused by medical insurance coverage for trastuzumab in Jiangsu, China. We present the following article in accordance with the MDAR reporting checklist (available at http://dx.doi.org/10.21037/tcr-20-3329).

\section{Methods}

\section{Study design}

This study was a retrospective, multicenter clinical study with follow-up data. It covered seven representative hospitals in seven cities in Jiangsu province. The study was based on data extracted from patients' hospital records in 2010, 2011, and 2013 to avoid interference. The participating hospitals are listed in Table S1. The study was conducted in accordance with the Declaration of Helsinki (as revised in 2013). The study was approved by the ethics committee of the First Affiliated Hospital with Nanjing Medical University (Jiangsu Province Hospital, No.: 2018SR-205). Informed consent form was signed by every patient.

\section{Patients}

This study included patients diagnosed with HER2positive EBC. Patients had to meet the following criteria for enrollment: aged $\geq 18$ and $\leq 85$ years; were diagnosed with HER2-positive breast cancer on the basis of a pathological report after breast surgery or core needle biopsy; had undergone surgery for breast cancer and received related treatments; had been staged lower than T4N3M0. Patients who had inflammatory breast cancer, developed metastatic disease, or had pre-invasive carcinoma were excluded. All patients included in the study met the HER2-positive diagnostic standard (either HER2 gene amplified by fluorescent in situ hybridization or 3+ by immunohistochemistry) according to the American Society of Clinical Oncology/College of American Pathologists 
clinical practice guidelines (12). The eligible patients were divided into two groups: the trastuzumab group and the non-trastuzumab group. The different characteristics between the two groups were analyzed.

\section{Data collection}

Data were extracted from the medical records. Telephone conversations were permitted if necessary. Demographic and clinical data (patient identifier, date of birth, height, weight, medical insurance status, menstruation status, heart disease history), diagnosis data (diagnostic date, surgical method, pathological type, pathological stage, receptor status), treatment data (whether neoadjuvant or adjuvant chemotherapy was received, whether trastuzumab was administered, the duration of trastuzumab treatment, whether radiotherapy was received, whether endocrine therapy was received), and prognostic data (date of death) were recorded for each patient.

\section{Overall survival analysis}

Overall survival analysis was performed to compare between women in the trastuzumab group and women in the nontrastuzumab group. The last follow-up was 31 January, 2020. Patients without mortality information were excluded.

\section{Stratified analysis}

Several studies have indicated that medical insurance status affects the use of trastuzumab (11,13-15). Trastuzumab entered the medical insurance dictionary of Jiangsu Healthcare Security Administration in 2012. In the current study, we explored whether medical insurance cover affected the use of trastuzumab. To accurately assess this, patients diagnosed with breast cancer in 2012 were excluded from the study.

\section{Statistical analysis}

All continuous variables were presented as the mean \pm standard deviation (SD). Levene's test was used to test the homogeneity of variance. Categorical data and proportions were analyzed using the $\chi^{2}$ or Fisher's exact test, whereas continuous variables were analyzed using the Student's $t$-test (normally distributed) or the Mann-Whitney $U$ test (non-normally distributed). The variables that attained a $\mathrm{P}$ value of $<0.10$ by univariate analysis were entered into a multivariate logistic regression model. Multivariate logistic regression analysis was used to identify the factors influencing the use of targeted therapy, and the results were expressed as odds ratio (OR) and $95 \%$ confidence interval (CI). The Kaplan-Meier method was used to identify the differences in overall survival between the trastuzumab group and the non-trastuzumab group. All statistical tests were 2 -sided, and $\mathrm{P}<0.05$ was considered statistically significant. SPSS 23.0 (IBM Corp., Armonk, NY) was used for the analyses.

\section{Results}

\section{Patients}

A total of 641 patients (mean age $51.01 \pm 10.79$ years) were enrolled in the study (Figure 1). All of them were diagnosed with HER2-positive EBC. Detailed baseline demographics for patients included in the analysis are presented in Table 1. Among all patients included, trastuzumab therapy was given to 214 (33.39\%) patients. Only 8 patients received trastuzumab as a neoadjuvant therapy. A total of 412 (64.27\%) patients had medical insurance.

\section{Univariate analysis of factors associated with trastuzumab use}

Univariate analysis revealed that patients who received trastuzumab therapy and those who did not receive trastuzumab therapy differed significantly in regard to the following factors: age, menstrual status, medical insurance, lymph node stage, and radiotherapy (all $\mathrm{P}<0.05$, Table 2). Patients who were younger, premenopausal, had medical insurance, had more lymph node involvement, and received radiotherapy were more likely to receive trastuzumab therapy. There was no significant difference between the two groups in body mass index (BMI), primary tumor stage, molecular subtype, or adjuvant endocrine therapy (Table 2).

\section{Multivariate logistic regression analysis}

Multivariate logistic regression analysis was performed to further identify the factors associated with the use of trastuzumab therapy. The factors that differed significantly in the univariate analysis were entered into the multivariate analysis. The details of the multivariate analysis are summarized in Table 3. The results showed that compared with patients who were older than 60 years, 


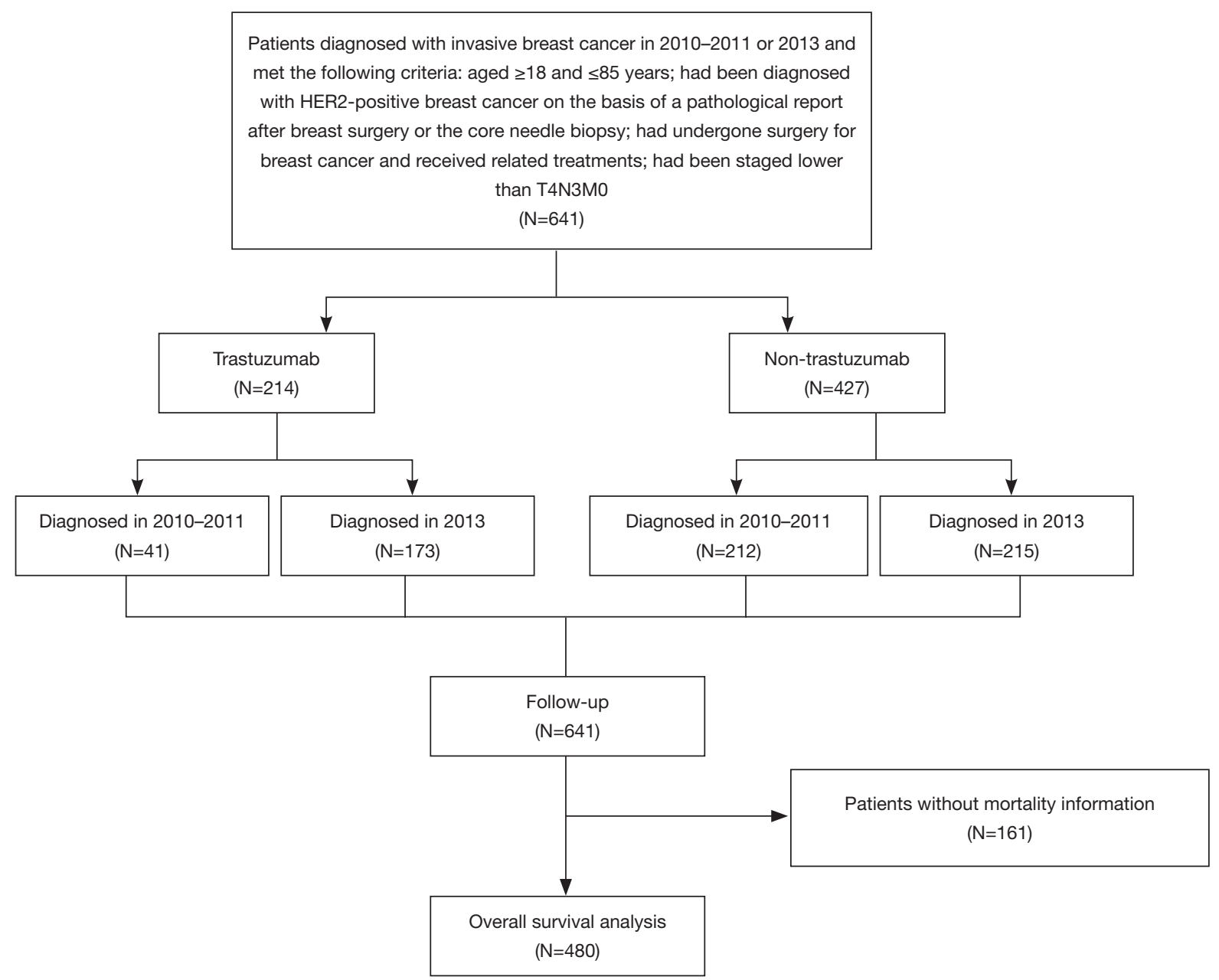

Figure 1 The flow chart of the patients included in the study.

patients who were younger than 35 years were more likely to receive trastuzumab therapy (OR: 3.095 ; $95 \%$ CI: 1.219-7.853). Medical insurance was strongly correlated with trastuzumab use (OR: 3.909; 95\% CI: 2.505-6.101). Adjuvant radiotherapy was also associated with higher use of trastuzumab therapy (OR: 2.700; 95\% CI: 1.630-4.472). Other factors, including lymph node stage and menopausal status, showed no significant association with trastuzumab therapy.

\section{Overall survival analysis}

A total of 161 patients without mortality information were excluded. The median follow-up time was 81.17 months in the non-trastuzumab group and 77.67 months in the trastuzumab group. The overall survival was significantly better in the trastuzumab group than in the nontrastuzumab group [hazard ratio (HR): 1.607; 95\% CI: 1.046-2.469; $\mathrm{P}=0.404$, Figure 2].

\section{Stratified analysis}

Trastuzumab entered the medical insurance dictionary of Jiangsu in 2012. Table 4 compares the demographic and clinical characteristics between patients who were diagnosed with HER2-positive EBC in 2010-2011 and those diagnosed in 2013. More patients received trastuzumab therapy in 2013 than in $2010-2011(\mathrm{P}<0.001)$. In addition, more patients diagnosed in 2013 were hormone receptor positive, and received adjuvant endocrine therapy and radiotherapy (all $\mathrm{P}<0.05$, Table 4). It should be noted that more patients had medical insurance and more patients were 
Table 1 Baseline demographic and clinical characteristics of the patients included in the analysis

\begin{tabular}{|c|c|}
\hline Parameter & Value $(\mathrm{N}=641)$ \\
\hline Age, y & $51.01 \pm 10.79$ \\
\hline$<35, \mathrm{n}(\%)$ & $47(7.33)$ \\
\hline $35-60, \mathrm{n}(\%)$ & $474(73.95)$ \\
\hline$>60, \mathrm{n}(\%)$ & $120(18.72)$ \\
\hline Body mass index (BMI), $\mathrm{kg} / \mathrm{m}^{2}$ & $23.19 \pm 2.46$ \\
\hline$<18.5, \mathrm{n}(\%)$ & $14(2.18)$ \\
\hline 18.5-23.9, n (\%) & $418(65.21)$ \\
\hline$\geq 24, \mathrm{n}(\%)$ & $209(32.61)$ \\
\hline \multicolumn{2}{|l|}{ Menstrual status, n (\%) } \\
\hline Premenopausal & $317(49.45)$ \\
\hline Postmenopausal & $324(50.55)$ \\
\hline \multicolumn{2}{|l|}{ Primary tumor stage, n (\%) } \\
\hline $\mathrm{T} 1$ & $320(49.92)$ \\
\hline $\mathrm{T} 2$ & $284(44.31)$ \\
\hline T3-4 & $37(5.77)$ \\
\hline \multicolumn{2}{|l|}{ Lymph node stage, n (\%) } \\
\hline NO & $359(56.01)$ \\
\hline N1 & $135(21.06)$ \\
\hline N2 & $70(10.92)$ \\
\hline N3 & $77(12.01)$ \\
\hline \multicolumn{2}{|l|}{ Molecular subtype, n (\%) } \\
\hline Hormone receptor positive & $296(46.18)$ \\
\hline Hormone receptor negative & $296(46.18)$ \\
\hline Missing & $49(7.64)$ \\
\hline \multicolumn{2}{|c|}{ Adjuvant endocrine therapy, n (\%) } \\
\hline Yes & $267(41.65)$ \\
\hline No & $330(51.48)$ \\
\hline Missing & $44(6.86)$ \\
\hline \multicolumn{2}{|l|}{ Radiotherapy, n (\%) } \\
\hline Yes & $201(31.36)$ \\
\hline No & $381(59.44)$ \\
\hline Missing & $59(9.20)$ \\
\hline \multicolumn{2}{|l|}{ Trastuzumab therapy, n (\%) } \\
\hline Yes & 214 (33.39) \\
\hline No & $427(66.61)$ \\
\hline \multicolumn{2}{|l|}{ Medical insurance, n (\%) } \\
\hline Yes & $412(64.27)$ \\
\hline No & $229(35.73)$ \\
\hline
\end{tabular}

diagnosed at an early tumor stage in 2013 than in 20102011, although this did not reach statistical significance.

\section{Discussion}

The current study was implemented in seven general hospitals across different cities in Jiangsu province. Trastuzumab use in these hospitals was the highest in Jiangsu province. In 2010-2011 and 2013, trastuzumab therapy was given to $33.39 \%$ of patients with EBC. The percentage of patients who received trastuzumab in this study was similar to another study implemented in China in 2013-2014, in which $29.8 \%$ of patients with HER2-positive EBC received trastuzumab treatment (11). However, the data was much lower than developed countries. Several studies have shown that $66-78.1 \%$ of patients received trastuzumab treatment in developed countries between 2005 and 2013 (14-18), though data from other developing countries is insufficient. Furthermore, the overall survival in our study was consistent with 2 large prospective clinical studies $(4,19)$.

In this study, several factors were found to be associated with trastuzumab therapy. The presence or absence of medical insurance was the strongest predictor of trastuzumab use. Patients who had medical insurance were 3.909 times more likely to use trastuzumab than those without medical insurance. Hospitals offered trastuzumab therapy to $42.96 \%$ of patients with medical insurance, whereas they provided trastuzumab to $16.16 \%$ of patients without medical insurance. There are two specific reasons behind this. On the one hand, medical insurance was limited to the urban population who had a higher income, so were more likely to afford trastuzumab. On the other hand, trastuzumab was covered by medical insurance from 2012, which greatly reduced the out-of-pocket costs of patients. Several studies also revealed that economic burden was the main barrier to the use of trastuzumab. In China, another study demonstrated that patients who resided in more prosperous provinces/cities (with higher incomes) or in provinces/cities which provided medical insurance for trastuzumab (Beijing, Jiangsu, and Ningxia) were more likely to be treated with trastuzumab (11). Other research has also shown that physicians can decide not to prescribe anti-HER2 therapies where such treatment has regulatory approval but is not funded or reimbursed. A survey of oncologists in the United States and emerging markets (Brazil, Mexico, Turkey, and Russia) showed that among the respondents who reported "not so often," "rarely," or 
Table 2 Univariate analysis of the factors associated with the use of trastuzumab therapy

\begin{tabular}{|c|c|c|c|}
\hline Parameter & Trastuzumab $(\mathrm{N}=214)$ & Non-trastuzumab (N=427) & $P$ value \\
\hline$<35, \mathrm{n}(\%)$ & $26(12.15)$ & $21(4.92)$ & 0.001 \\
\hline $35-60, \mathrm{n}(\%)$ & $159(74.30)$ & $315(73.77)$ & \\
\hline$>60, \mathrm{n}(\%)$ & $29(13.55)$ & $91(21.31)$ & \\
\hline$<18.5, \mathrm{n}(\%)$ & $3(1.40)$ & $11(2.58)$ & 0.578 \\
\hline 18.5-23.9, n (\%) & $143(66.82)$ & $275(64.40)$ & \\
\hline$\geq 24, \mathrm{n}(\%)$ & 68 (31.78) & $141(33.02)$ & \\
\hline Menstrual status, n (\%) & & & 0.001 \\
\hline Primary tumor stage, n (\%) & & & 0.688 \\
\hline T1 & $107(50.00)$ & $213(49.88)$ & \\
\hline $\mathrm{T} 2$ & $97(45.33)$ & $187(43.79)$ & \\
\hline T3-4 & $10(4.67)$ & $27(6.32)$ & \\
\hline Lymph node stage, n (\%) & & & 0.017 \\
\hline NO & $102(47.66)$ & $257(60.19)$ & \\
\hline N1 & $50(23.36)$ & 85 (19.91) & \\
\hline N2 & 28 (13.08) & $42(9.84)$ & \\
\hline Adjuvant endocrine therapy, n (\%) & & & 0.261 \\
\hline Yes & $97(48.26)$ & $170(42.93)$ & \\
\hline No & $104(51.74)$ & $226(57.07)$ & \\
\hline Radiotherapy, n (\%) & & & $<0.001$ \\
\hline Yes & $100(51.28)$ & $101(26.10)$ & \\
\hline No & 95 (48.72) & $286(73.90)$ & \\
\hline Medical insurance, n (\%) & & & $<0.001$ \\
\hline Yes & $177(82.71)$ & $235(55.04)$ & \\
\hline No & 37 (17.29) & $192(44.96)$ & \\
\hline
\end{tabular}


Table 3 Multivariate analysis of the factors associated with the use of trastuzumab therapy

\begin{tabular}{lcc}
\hline Parameter & Odds ratio $(95 \% \mathrm{Cl})$ & P value \\
\hline Age, y & 1.000 & \\
$>60$ & $3.095(1.219-7.853)$ & 0.017 \\
$<35$ & $1.109(0.622-1.979)$ & 0.726 \\
$35-60$ & 1.000 & \\
Lymph node stage & $1.064(0.547-2.068)$ & 0.855 \\
N0 & $0.925(0.478-1.788)$ & 0.816 \\
N1 & $0.870(0.401-1.847)$ & 0.718 \\
N2 & 1.000 & \\
N3 & 1.000 & $<0.001$ \\
Menstrual status & $0.818(0.530-1.261)$ & 0.363 \\
Premenopausal & & \\
Postmenopausal & 1.000 & \\
Radiotherapy & $2.700(1.630-4.472)$ & $<0.001$ \\
No & & \\
Yes & & \\
Medical insurance & & \\
No & & \\
Yes & & \\
\hline
\end{tabular}

"never" prescribing trastuzumab, between $37 \%$ and $49 \%$ considered the lack of drug funding a barrier to the use of trastuzumab (13). Of the 151 physicians participating in TEACH, an international survey conducted in 2011, $27 \%$ of respondents reported at least 1 instance within the previous year in which adjuvant trastuzumab was recommended to a patient who ultimately did not receive it, citing cost as the reason for withholding treatment. Furthermore, cost was more often taken into account by physicians in low- and middle-income countries (73\%) than in high-income countries $(7 \%, \mathrm{P}<0.0001)$ as a reason for withholding adjuvant trastuzumab (20).

There was a significant difference in the percentage of patients who received trastuzumab between 2013 and 2010-2011 (44.59\% in 2013 versus $16.21 \%$ in $2010-2011$, $\mathrm{P}<0.001)$. It should be noted that in 2013 , more patients had medical insurance $(67.01 \%$ in 2013 versus $60.08 \%$ in 2010-2011), although this did not reach statistical significance $(\mathrm{P}=0.073)$. This trend was certainly due to the health-care reform in China. In April 2009, China unveiled a huge and complex heath reform plan that pledged to provide all citizens with equal access to basic health care with reasonable quality and sufficient financial risk protection by 2020 (9). It emphasized insurance expansion in its first phase. Over the past 10 years, substantial progress

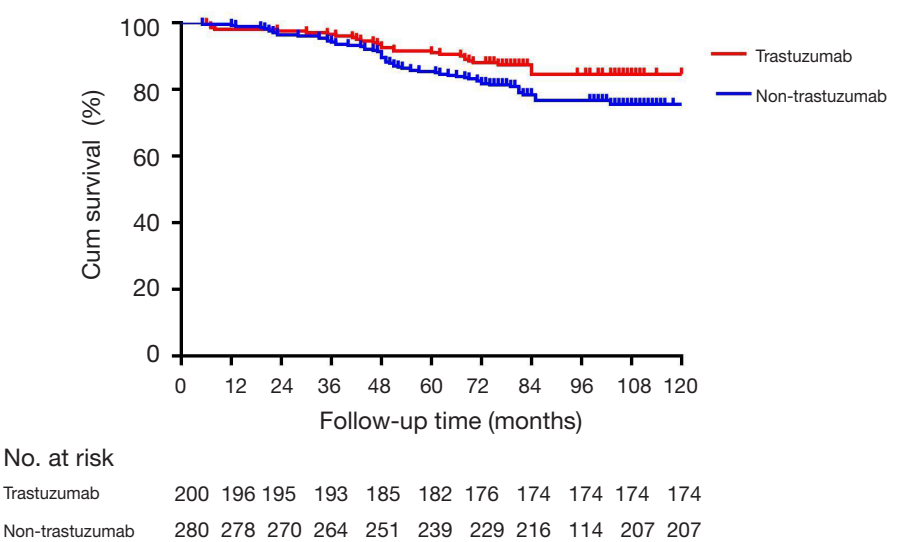

Figure 2 Overall survival between the trastuzumab group and the non-trastuzumab group calculated using the Kaplan-Meier method. HR: $1.607,95 \%$ CI: $1.046-2.469, \mathrm{P}=0.040$. 
Table 4 Comparison of trastuzumab use between patients who were diagnosed with early-stage breast cancer (EBC) in 2010-2011 and in 2013

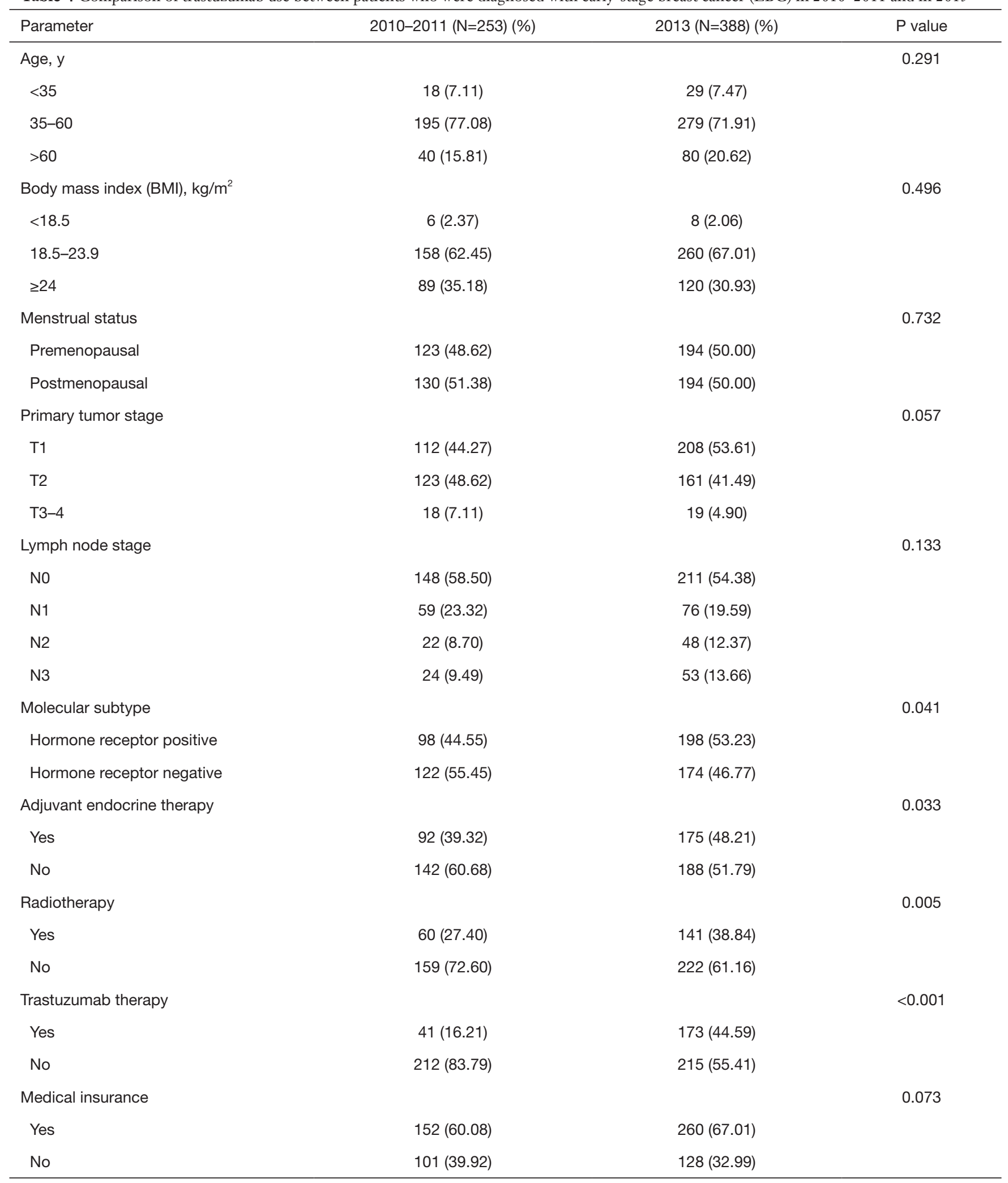


was made in improving equal access to care and enhancing financial protection (21). In Jiangsu province, the insurance expansion together with the medical insurance coverage for trastuzumab greatly improved the rate of adjuvant trastuzumab use.

We also observed a trend towards more patients diagnosed with an earlier tumor stage in $2013(\mathrm{P}=0.057)$. This may be the result of enhanced breast cancer screening. First attempted in 2005 , a national screening program for breast cancer set a goal of screening 1,000,000 women with both mammography and ultrasound, but was terminated because of a lack of funding and concerns about falsepositive diagnosis (22). In 2009-2011, a "two-cancer" (breast cancer and cervical cancer) screening program was implemented for women aged 35-59 years in rural China and in some urban areas (23). Therefore, more accessible health services were provided to Chinese women, and breast screening has been taken more seriously since then. The results in our study reflected this phenomenon, particularly that patients younger than 35 years old were more likely to use trastuzumab therapy. In fact, several studies have demonstrated that trastuzumab was withheld from patients because of advanced age $(24,25)$.

The strength of this study is that it is based on real-world data. It is also the first study, to the best of our knowledge, to explore the significance of health insurance policy in China on clinical practice. However, as a retrospective study, the current research has some limitations. Data were missing for many of the variables analyzed in some of the patients, which may bring information bais. In addition, the characteristics of patients in the trastuzumab group and those in the non-trastuzumab group were not evenly distributed. The unevenly distributed characteristics does may make it unreliable to directly analysis factors associated with the use of trastuzumab therapy. However we also took all variables with $\mathrm{P}$ value $<0.10$, including these potential confounders, from univariate analysis into the multivariate logistic regression model to identify factors independent associated with the targeted therapy. And we finally used the outcomes of the multivariate analysis to identify influencing factors. Therefore, large prospective randomized clinical trials are warranted to further verify the results of our study.

In conclusion, this study analyzed the factors affecting the use of trastuzumab as an adjuvant therapy for HER2positive breast cancer patients in China. The results showed that the percentage of patients who received trastuzumab in China was lower than developed countries. Patients who had medical insurance were more likely to use trastuzumab than those without medical insurance. With the development of health-care reform in China, more patients could afford the targeted therapy essential for tumor management. Considering all this evidence, the health insurance policy in China has made great progress towards improving access for breast cancer patients who require trastuzumab therapy.

\section{Acknowledgments}

Funding: This study was financially supported by the Nanjing Medical University Education Development Foundation (JZ233490201802) and the Project of Philosophy and Social Science Research in Colleges and Universities in Jiangsu Province (2020SJA0292).

\section{Footnote}

Reporting Checklist: The authors have completed the MDAR reporting checklist. Available at http://dx.doi.org/10.21037/ tcr-20-3329

Data Sharing Statement: Available at http://dx.doi. org/10.21037/tcr-20-3329

Conflicts of Interest: All authors have completed the ICMJE uniform disclosure form (available at http://dx.doi. org/10.21037/tcr-20-3329). The authors have no conflicts of interest to declare.

Ethical Statement: The authors are accountable for all aspects of the work in ensuring that questions related to the accuracy or integrity of any part of the work are appropriately investigated and resolved. The study was conducted in accordance with the Declaration of Helsinki (as revised in 2013). The study was approved by the ethics committee of the First Affiliated Hospital with Nanjing Medical University (Jiangsu Province Hospital, No.: 2018SR-205). Informed consent form was signed by every patient.

Open Access Statement: This is an Open Access article distributed in accordance with the Creative Commons Attribution-NonCommercial-NoDerivs 4.0 International License (CC BY-NC-ND 4.0), which permits the noncommercial replication and distribution of the article with the strict proviso that no changes or edits are made and the original work is properly cited (including links to both the formal publication through the relevant DOI and the license). 
See: https://creativecommons.org/licenses/by-nc-nd/4.0/.

\section{References}

1. Bray F, Ferlay J, Soerjomataram I, et al. Global cancer statistics 2018: GLOBOCAN estimates of incidence and mortality worldwide for 36 cancers in 185 countries. CA Cancer J Clin 2018;68:394-424.

2. Waks AG, Winer EP. Breast Cancer Treatment: A Review. JAMA 2019;321:288-300.

3. Piccart-Gebhart MJ, Procter M, Leyland-Jones B, et al. Trastuzumab after adjuvant chemotherapy in HER2positive breast cancer. N Engl J Med 2005;353:1659-72.

4. Perez EA, Romond EH, Suman VJ, et al. Trastuzumab plus adjuvant chemotherapy for human epidermal growth factor receptor 2-positive breast cancer: planned joint analysis of overall survival from NSABP B-31 and NCCTG N9831. J Clin Oncol 2014;32:3744-52.

5. National Comprehensive Cancer Network. NCCN Clinical Practice Guidelines in Oncology: breast cancer, version 1.2019. Available online: https://www.nccn.org/ 2019 Accessed: March 14, 2019.

6. Cardoso F, Kyriakides S, Ohno S, et al. Early breast cancer: ESMO Clinical Practice Guidelines for diagnosis, treatment and follow-up. Ann Oncol 2019;30:1674.

7. Breast Cancer Guidelines of Chinese Society of Clinical Oncology (CSCO), 2019. Available online: http://www. csco.org.cn

8. Blackwell K, Gligorov J, Jacobs I, et al. The Global Need for a Trastuzumab Biosimilar for Patients With HER2-Positive Breast Cancer. Clin Breast Cancer 2018;18:95-113.

9. Yip WC, Hsiao WC, Chen W, et al. Early appraisal of China's huge and complex health-care reforms. Lancet 2012;379:833-42.

10. Li J, Wang S, Wang Y, et al. Disparities of Trastuzumab Use in Resource-Limited or Resource-Abundant Regions and Its Survival Benefit on HER2 Positive Breast Cancer: A Real-World Study from China. Oncologist 2017;22:1333-8.

11. Li J, Shao Z, Xu B, et al. Use of trastuzumab as an adjuvant/neoadjuvant therapy in patients with HER2positive breast cancer in China: The Nvwa study. Medicine (Baltimore) 2018;97:e10350.

12. Wolff AC, Hammond MEH, Allison KH, et al. Human Epidermal Growth Factor Receptor 2 Testing in Breast Cancer: American Society of Clinical Oncology/College of American Pathologists Clinical Practice Guideline
Focused Update. J Clin Oncol 2018;36:2105-22.

13. Lammers P, Criscitiello C, Curigliano G, et al. Barriers to the Use of Trastuzumab for HER2+ Breast Cancer and the Potential Impact of Biosimilars: A Physician Survey in the United States and Emerging Markets. Pharmaceuticals (Basel) 2014;7:943-53.

14. Wilking $\mathrm{U}$, Jönsson B, Wilking $\mathrm{N}$, et al. Trastuzumab use in breast cancer patients in the six Health Care Regions in Sweden. Acta Oncol 2010;49:844-50.

15. Lawrenson R, Lao C, Campbell I, et al. The use of trastuzumab in New Zealand women with breast cancer. Asia Pac J Clin Oncol 2018;14:e152-e160.

16. Seferina SC, Lobbezoo DJ, de Boer M, et al. Real-Life Use and Effectiveness of Adjuvant Trastuzumab in Early Breast Cancer Patients: A Study of the Southeast Netherlands Breast Cancer Consortium. Oncologist 2015;20:856-63.

17. Webster RM, Abraham J, Palaniappan N, et al. Exploring the use and impact of adjuvant trastuzumab for HER2positive breast cancer patients in a large UK cancer network. Do the results of international clinical trials translate into a similar benefit for patients in South East Wales? Br J Cancer 2012;106:32-8.

18. DaCosta Byfield S, Buck PO, Blauer-Peterson C, et al. ReCAP: Treatment Patterns and Cost of Care Associated With Initial Therapy Among Patients Diagnosed With Operable Early-Stage Human Epidermal Growth Factor Receptor 2-Overexpressed Breast Cancer in the United States: A Real-World Retrospective Study. J Oncol Pract 2016;12:159-67.

19. Cameron D, Piccart-Gebhart MJ, Gelber RD, et al. 11 years' follow-up of trastuzumab after adjuvant chemotherapy in HER2-positive early breast cancer: final analysis of the HERceptin Adjuvant (HERA) trial. Lancet 2017;389:1195-205.

20. Chavarri-Guerra Y, St Louis J, Bukowski A, et al. Real world patterns of care in HER2-overexpressing breast cancer: Results of a survey of TEACH clinical trial investigators in 2011. Breast 2017;31:197-201.

21. Yip W, Fu H, Chen AT, et al. 10 years of health-care reform in China: progress and gaps in Universal Health Coverage. Lancet 2019;394:1192-204.

22. Fan L, Strasser-Weippl K, Li JJ, et al. Breast cancer in China. Lancet Oncol 2014;15:e279-89.

23. Zuo TT, Zheng RS, Zeng HM, et al. Female breast cancer incidence and mortality in China, 2013. Thorac Cancer 2017;8:214-8.

24. Coulson SG, Kumar VS, Manifold IM, et al. Review of testing and use of adjuvant trastuzumab across a cancer 
network--are we treating the right patients? Clin Oncol (R Coll Radiol) 2010;22:289-93.

25. Stenehjem DD, Yoo M, Unni SK, et al. Assessment of HER2 testing patterns, HER2 + disease, and the utilization

Cite this article as: Xia Y, Zheng M, Zhan X, Liu Y, Cao S, Shao Q, Meng D, Jin L, Xu L, Yi T, Xie H, Wang S. The use of trastuzumab affected by health insurance policy in Jiangsu Province of China. Transl Cancer Res 2021;10(1):509-519. doi: $10.21037 /$ tcr-20-3329 of HER2-directed therapy in early breast cancer. Breast Cancer (Dove Med Press) 2014;6:169-77.

(English Language Editor: C. Betlazar-Maseh) 